\title{
Over-expression of ITK in metastatic human breast cancer.
}

Shahan Mamoor, $\mathrm{MS}^{1}$

${ }^{1}$ shahanmamoor@gmail.com

East Islip, NY USA

Metastasis to the brain is a clinical problem in patients with breast cancer ${ }^{1-3}$. We mined published microarray data ${ }^{4,5}$ to compare primary and metastatic tumor transcriptomes for the discovery of genes associated with metastasis to the lymph nodes and the brain in humans with metastatic breast cancer. We found that the IL-2-inducible T-cell kinase, encoded by ITK, was among the genes whose expression was most different in the lymph node metastases of patients with metastatic breast cancer as compared to primary tumors of the breast. ITK mRNA was present at increased quantities in lymph node metastases as compared to primary tumors of the breast. ( overall survival. Modulation of ITK expression may be relevant to the biology by which tumor cells metastasize from the breast to the brain in humans with metastatic breast cancer. Inhibition of ITK represents a potential therapeutic approach in halting progression of lymph node metastatic breast cancer.

Keywords: breast cancer, metastasis, brain metastases, central nervous system metastases, the IL-2-inducible T-cell kinase, ITK, systems biology of breast cancer, targeted therapeutics in breast cancer. 
One report described a $34 \%$ incidence of central nervous system metastases in patients compared administration of either neratinib or trastuzumab in conjunction with paclitaxel demonstrated that in a randomized, controlled setting, in breast cancer patients treated with neratinib, not only was the incidence of central nervous system recurrence significantly lower, the time to central nervous system metastasis was significantly delayed as compared to patients administered trastuzumab ${ }^{6}$. The alarmingly high rate of central nervous system metastasis described, as well as data, both anecdotal ${ }^{2}$ and from a randomized, controlled setting ${ }^{6}$ illustrating that treatment with trastuzumab may be associated with these events demands an enhanced understanding of the transcriptional makeup of brain metastatic tissues to support identification of therapeutic targets, whether they are treatment related or not. We performed a global comparative analysis of lymph node and brain metastatic tumor tissues in patients with metastatic breast cancer, comparing to primary tumors and normal breast tissues, respectively ${ }^{4,5}$. We discovered transcriptome-wide differential expression of the gene encoding the IL-2-inducible T-cell kinase, ITK, in lymph node and brain metastatic tissues of patients with metastatic breast cancer.

\section{$\underline{\text { Methods }}$}

We used datasets GSE $10893^{4}$ and GSE52604 5 for this global differential gene expression analysis of brain metastatic breast cancer in conjunction with GEO2R. GSE10893 was generated using Agilent-011521 Human 1A Microarray G4110A technology with $n=71$ primary breast tumors and $n=7$ lymph node metastases from patients with breast cancer; analysis was performed using platform GPL887. GSE52604 was generated using Agilent-014850 Whole Human Genome Microarray $4 \mathrm{x} 44 \mathrm{~K}$ G4112F with $n=10$ normal breast tissues and $n=35$ brain metastases from patients with breast cancer; analysis was performed using platform GPL6480. The Benjamini and Hochberg method of $p$-value adjustment was used for ranking of differential expression but raw $p$-values were used to assess statistical significance of global differential expression.

Log-transformation of data was auto-detected, and the NCBI generated category of platform annotation was used. A statistical test was performed to evaluate whether ITK gene expression was significantly different between primary tumors of the breast and lymph node metastases in humans with breast cancer using a two-tailed t-test. For Kaplan-Meier survival analysis, we used the Kaplan-Meier plotter online tool ${ }^{7}$ for correlation of ITK mRNA expression levels with overall survival in $n=1402$ breast cancer patients.

\section{$\underline{\text { Results }}$}

We performed global comparative transcriptome analysis of metastatic tumor tissues of patients with metastatic breast cancer using published microarray data ${ }^{4,5}$ to describe the transcriptional landscape of metastasis in human breast cancer in an unbiased fashion and for the discovery of novel therapeutic targets.

\section{ITK is differentially expressed in the lymph node metastases of patients with metastatic breast cancer.}

Through blind, systems-level analysis of published microarray data ${ }^{4}$, we identified the 
IL-2-inducible T-cell kinase, encoded by ITK, as a differentially expressed gene in the lymph node metastatic tissues of humans with breast cancer (Table 1). When sorting each of the genes expressed in lymph node metastases based on significance of difference as compared to primary tumors of the breast in patients with breast cancer, ITK ranked 34 out of 18034 total transcripts (Chart 1), equating to $99.8 \%$ differential expression. Differential expression of ITK in the lymph node metastases of patients with metastatic breast cancer was statistically significant (Chart 1; $p=1.39 \mathrm{E}-04)$.

Differential gene expression analyses that utilize primary and metastatic tumor tissues to discover genes associated with metastasis can be challenging due to the relatively heterogenous cellular composition and constitution of the tumor as compared to normal, untransformed (benign) tissues. Thus, to attempt to validate differential transcriptome-wide differential expression of ITK in human metastatic breast cancer, we queried a second microarray dataset ${ }^{5}$, here comparing normal breast tissues to brain metastases. Again, we identified ITK as a differentially expressed gene in the metastatic tissues of patients with breast cancer (Chart 2). When sorting each of the genes expressed in brain metastases based on significance of difference as compared to normal breast tissues, ITK ranked 10693 out of 41093 total transcripts (Chart 2), equating to $74.0 \%$ differential expression. Differential expression of ITK in the brain metastases of patients with metastatic breast cancer was statistically significant (Chart $2 ; p=6.72 \mathrm{E}-03$ ). Thus, differential expression of ITK, transcriptome-wide, in the metastatic tissues of women with metastatic breast cancer was conserved across two independent microarray datasets, both when compared to benign and transformed breast tissues.

\section{ITK is expressed at higher levels in the lymph node metastases of patients with metastatic} breast cancer.

We obtained exact mRNA expression levels for ITK, in primary tumors of the breast and in lymph node metastases of patients with metastatic breast cancer to determine direction and statistical significance of change in ITK expression in lymph node metastatic tissues. ITK was expressed at higher levels in the lymph node metastases of patients with breast cancer as compared to primary tumors of the breast, and this difference was statistically significant (Figure $1 ; p=0.000172)$.

\section{ITK expression is significantly correlated with survival outcomes in human breast cancer.}

We performed Kaplan-Meier survival analysis ${ }^{8}$ in $n=1402$ breast cancer patients in total, to evaluate whether ITK tumor expression was correlated with survival outcomes in breast cancer. We observed a statistically significant correlation between primary tumor expression of ITK and overall survival (OS) in patients with breast cancer (Figure 2). Patients whose primary tumors expressed low levels of ITK possessed median OS of 72.2 months, while patients whose tumors expressed high levels of ITK possessed median OS of 138 months. This difference in OS based on ITK tumor expression in patients with breast cancer was statistically significant (Figure 2, Chart 3; logrank $p$-value: 0.00012; hazard ratio: $0.65(0.53-0.81))$. 
Thus, by mining published microarray data ${ }^{4,5}$ in an unbiased and systematic fashion, we identified the IL-2-inducible T-cell kinase, encoded by ITK, as among the genes whose expression was most different, transcriptome-wide, in the lymph node and brain metastases of tissues, respectively; we observed significantly increased expression of ITK in lymph node metastases as compared to primary tumors of the breast. Further, we found a significant correlation between ITK expression and patient survival outcomes, as overall survival was significantly greater in patients whose primary tumors expressed higher levels of ITK as compared to patients whose primary tumors expressed lower levels of ITK.

\section{Discussion}

We provided evidence here that the IL-2-inducible T-cell kinase, encoded by ITK, is among the genes whose expression is most different in the lymph node and brain metastases of patients with metastatic breast cancer, that ITK mRNA is present at significantly increased quantities in lymph node metastatic tissues as compared to primary tumors of the breast, and that primary tumor ITK expression is significantly correlated with patient survival outcomes in human breast cancer. Evaluation of the effects of genetic depletion of ITK in mouse models of metastatic breast cancer on metastasis to the central nervous system is merited. While we found that higher primary tumor expression of ITK was correlated with enhanced patient survival, patient survival outcomes based on primary tumor expression do not necessarily correlate with phenotypic consequence of inhibition. Moreover, the survival data we presented here is based on expression of ITK in primary tumors, not the lymph node metastatic tissues, in which we found significantly increased expression of ITK. An inhibitor of ITK, ibrutinib, is available for use in the clinic ${ }^{8}$. Modulation of ITK expression may be relevant to the processes by which breast cancer cells exit the breast, enter the vasculature and/or lymphatics, reside in the lymph nodes, evade immune clearance, breach the blood-brain barrier and colonize the brain. Inhibition of ITK represents a potential therapeutic approach in halting progression of lymph node metastatic breast cancer. 


\section{References}

1. Lin, N.U., Amiri-Kordestani, L., Palmieri, D., Liewehr, D.J. and Steeg, P.S., 2013. CNS metastases in breast cancer: old challenge, new frontiers.

2. Bendell, J.C., Domchek, S.M., Burstein, H.J., Harris, L., Younger, J., Kuter, I., Bunnell, C., Rue, M., Gelman, R. and Winer, E., 2003. Central nervous system metastases in women who receive trastuzumab-based therapy for metastatic breast carcinoma. Cancer, 97(12), pp.2972-2977.

3. Tsukada, Y., Fouad, A., Pickren, J.W. and Lane, W.W., 1983. Central nervous system metastasis from breast carcinoma autopsy study. Cancer, 52(12), pp.2349-2354.

4. Weigman, V.J., Chao, H.H., Shabalin, A.A., He, X., Parker, J.S., Nordgard, S.H., Grushko, T., Huo, D., Nwachukwu, C., Nobel, A. and Kristensen, V.N., 2012. Basal-like Breast cancer DNA copy number losses identify genes involved in genomic instability, response to therapy, and patient survival. Breast cancer research and treatment, 133(3), pp.865-880.

5. Salhia, B., Kiefer, J., Ross, J.T., Metapally, R., Martinez, R.A., Johnson, K.N., DiPerna, D.M., Paquette, K.M., Jung, S., Nasser, S. and Wallstrom, G., 2014. Integrated genomic and epigenomic analysis of breast cancer brain metastasis. Plos one, 9(1), p.e85448.

6. Awada, A., Colomer, R., Inoue, K., Bondarenko, I., Badwe, R.A., Demetriou, G., Lee, S.C., Mehta, A.O., Kim, S.B., Bachelot, T. and Goswami, C., 2016. Neratinib plus paclitaxel vs trastuzumab plus paclitaxel in previously untreated metastatic ERBB2-positive breast cancer: the NEfERT-T randomized clinical trial. JAMA oncology, 2(12), pp.1557-1564.

7. Györffy, B., Lanczky, A., Eklund, A.C., Denkert, C., Budczies, J., Li, Q. and Szallasi, Z., 2010. An online survival analysis tool to rapidly assess the effect of 22,277 genes on breast cancer prognosis using microarray data of 1,809 patients. Breast cancer research and treatment, 123(3), pp.725-731.

8. Dubovsky, J.A., Beckwith, K.A., Natarajan, G., Woyach, J.A., Jaglowski, S., Zhong, Y., Hessler, J.D., Liu, T.M., Chang, B.Y., Larkin, K.M. and Stefanovski, M.R., 2013. Ibrutinib is an irreversible molecular inhibitor of ITK driving a Th1-selective pressure in T lymphocytes. Blood, $122(15)$, pp.2539-2549. 


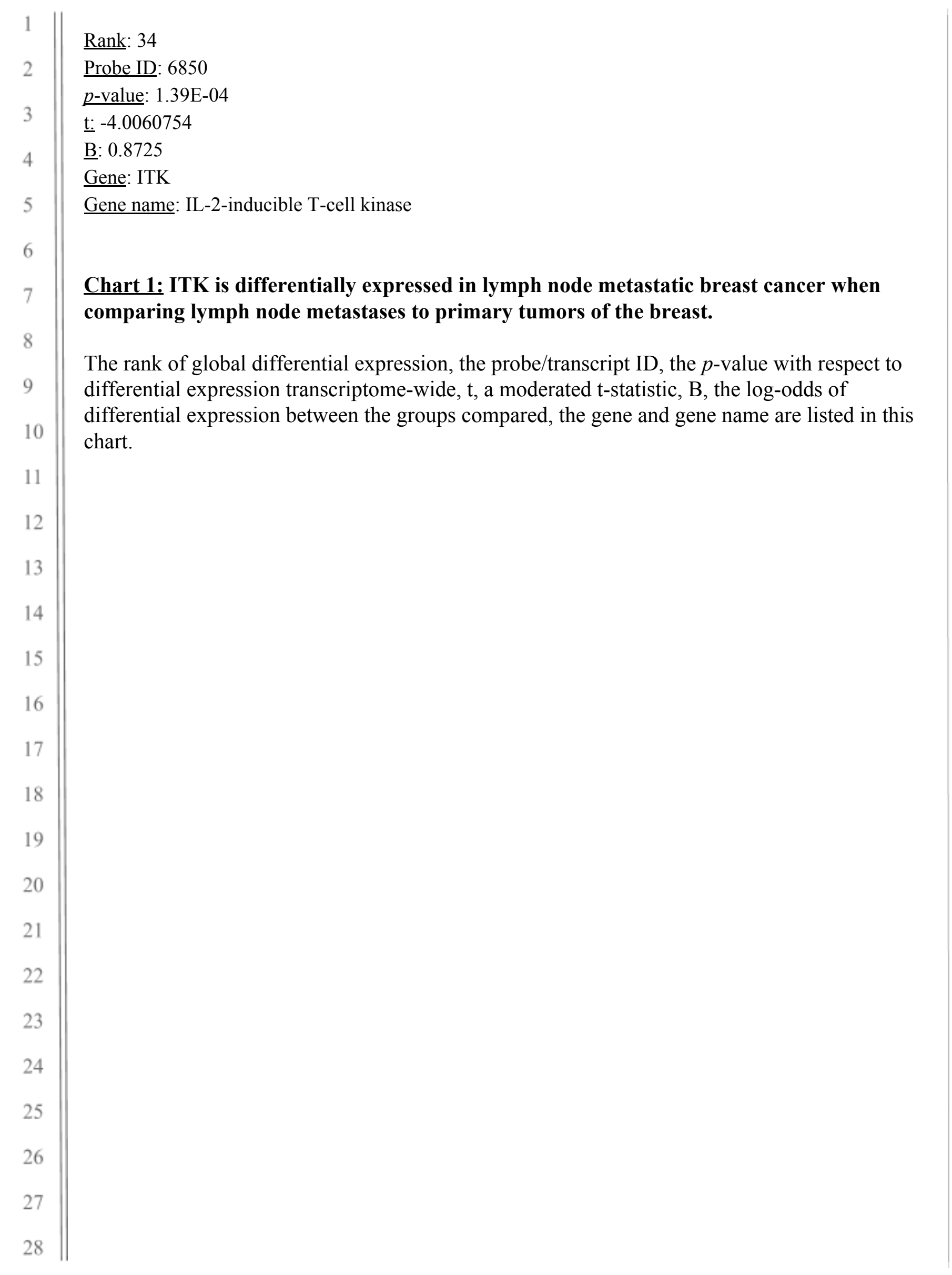




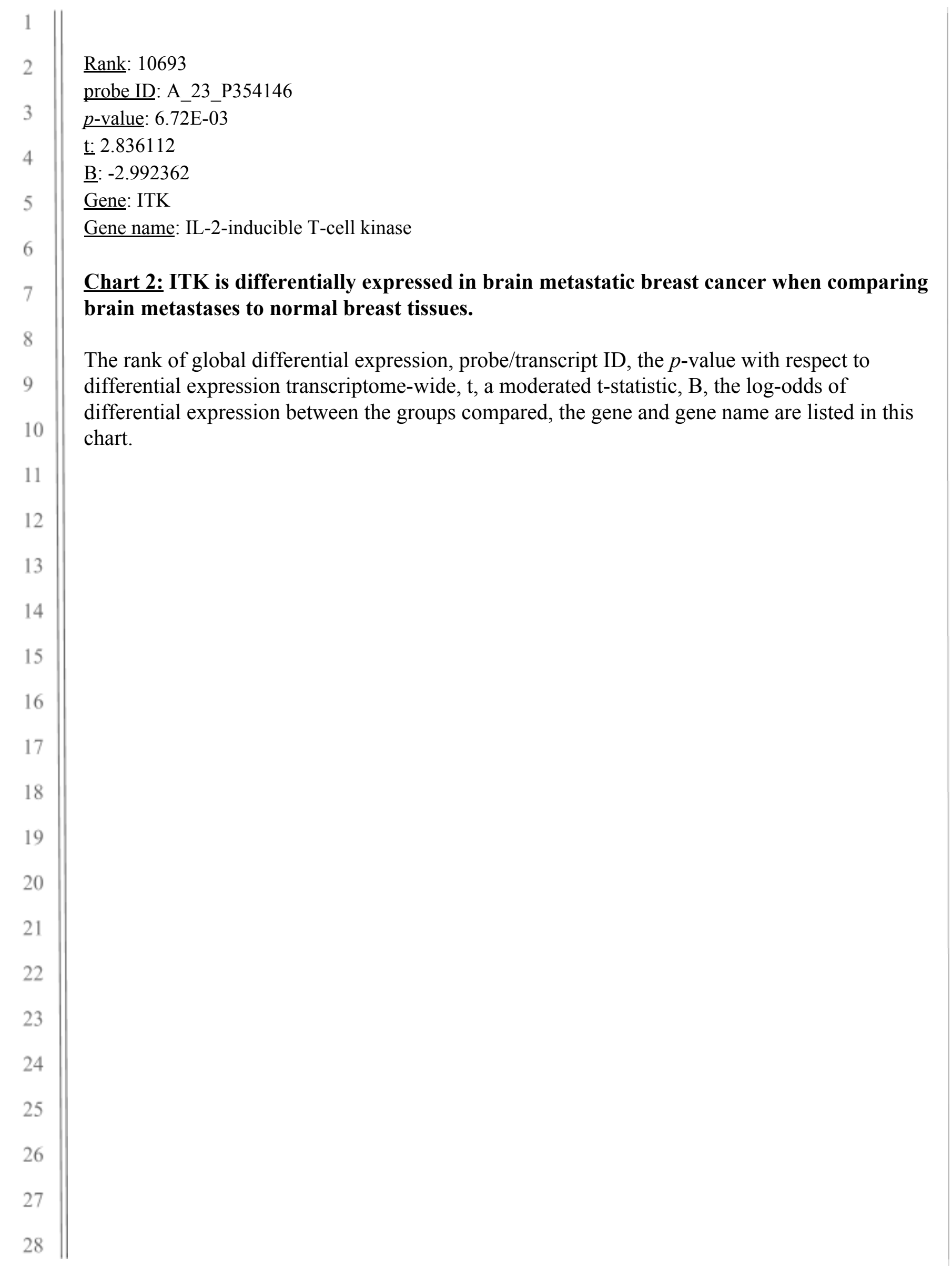




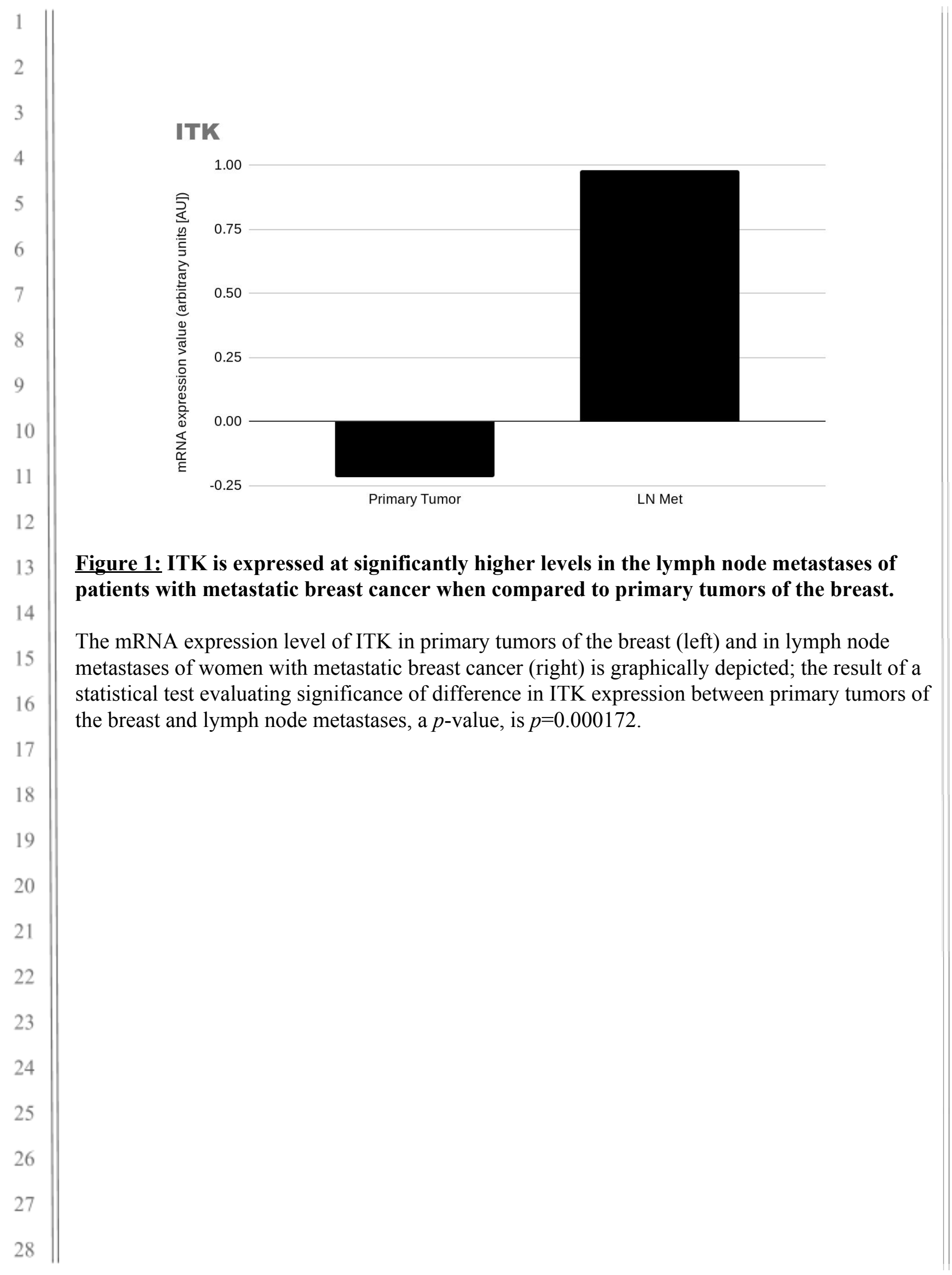




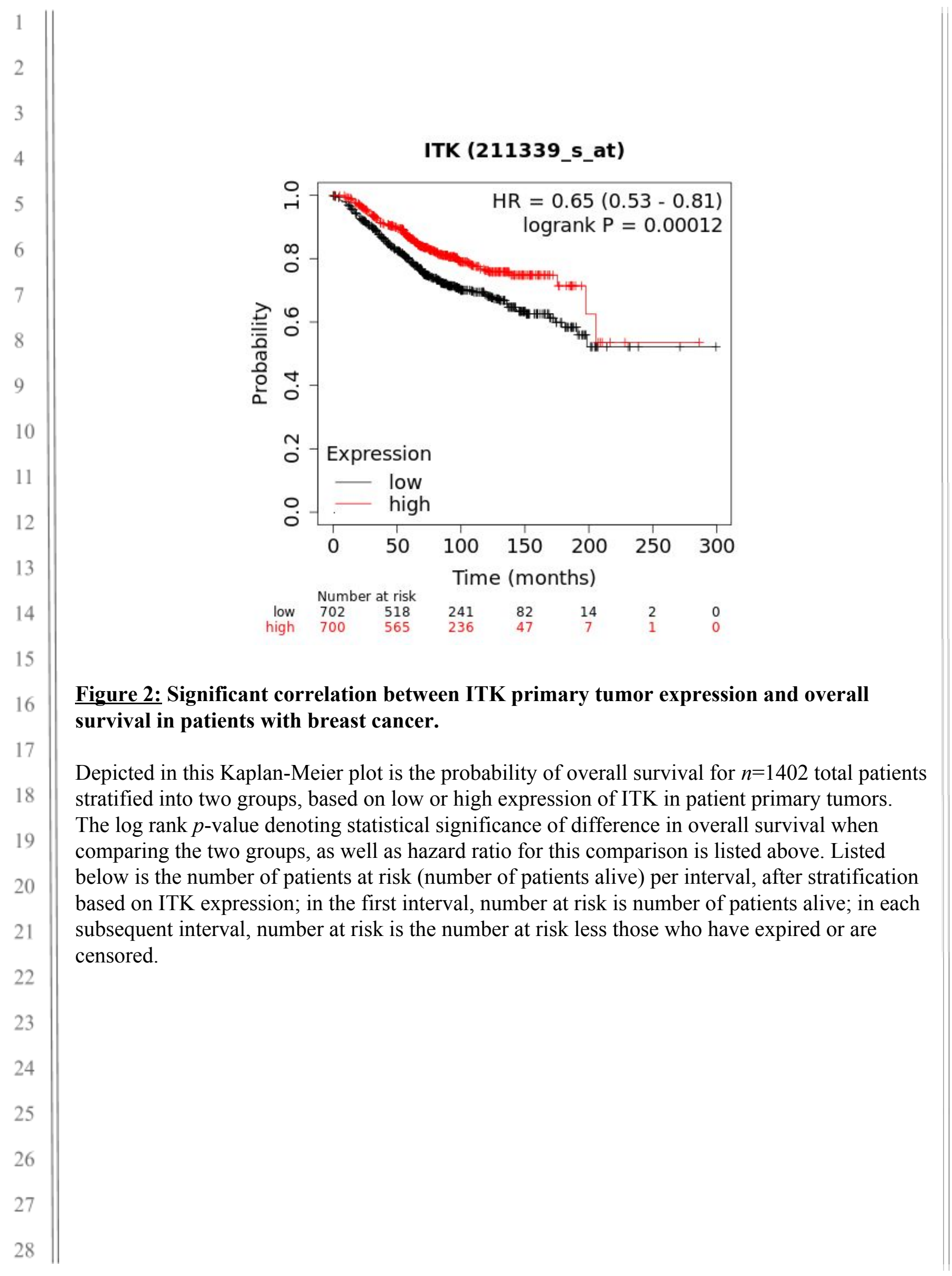




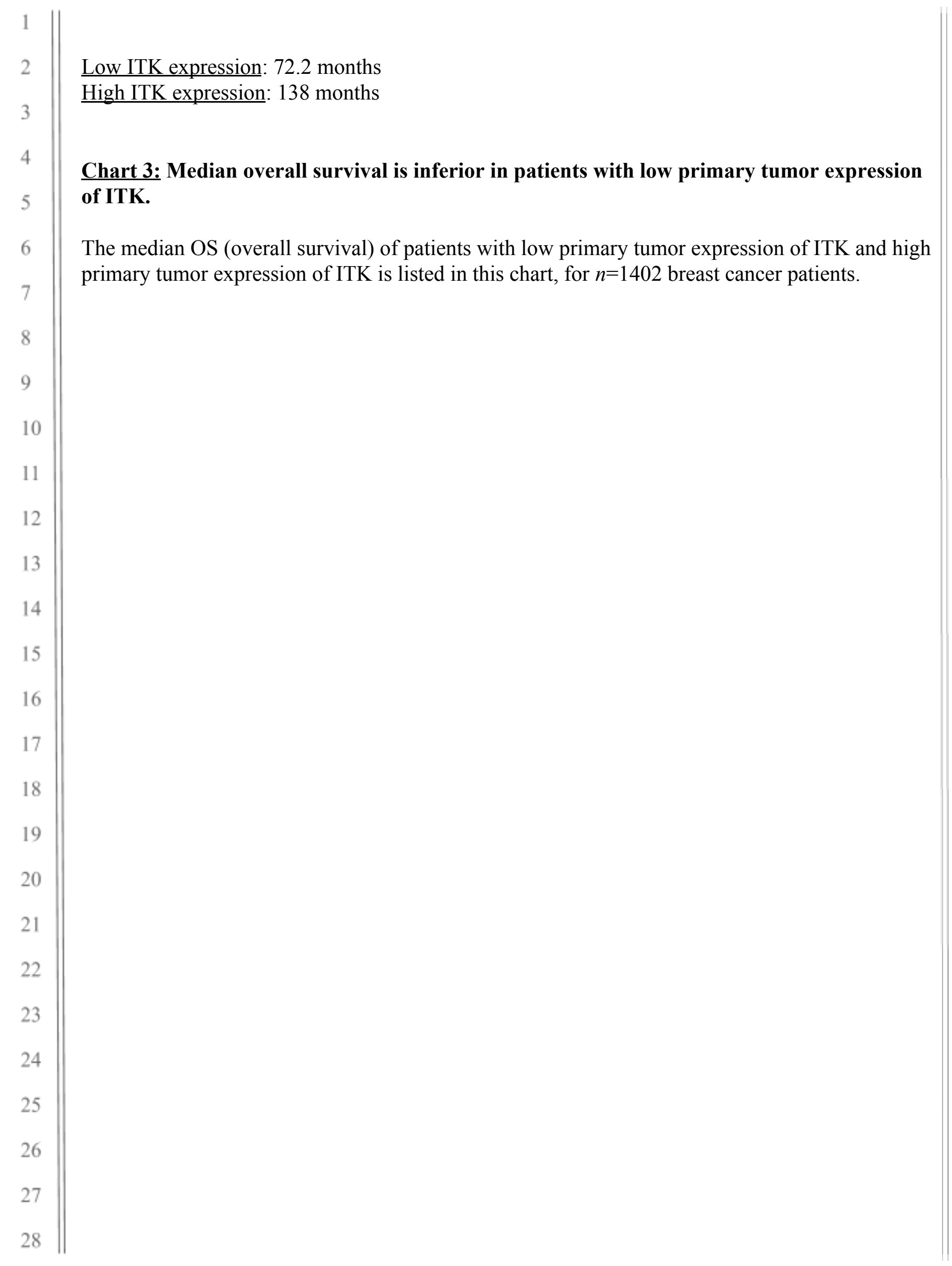

